\title{
Thoracic radiotherapy in small cell lung cancer-a narrative review
}

\author{
Antonin Levy ${ }^{1,2,3}$, Angela Botticella ${ }^{1}$, Cécile Le Péchoux ${ }^{1}$, Corinne Faivre-Finn ${ }^{4,5}$ \\ ${ }^{1}$ Department of Radiation Oncology, Institut d'Oncologie Thoracique (IOT), Gustave Roussy, Villejuif, France; ${ }^{2}$ Univ Paris Sud, Université Paris- \\ Saclay, Le Kremlin-Bicêtre, France; ${ }^{3}$ INSERM U1030, Molecular Radiotherapy, Gustave Roussy, Université Paris-Saclay, Villejuif, France; ${ }^{4}$ Division \\ of Cancer Sciences, School of Medical Sciences, Faculty of Biology, Medicine and Health, University of Manchester, Manchester, UK; ${ }^{5}$ The Christie \\ NHS Foundation Trust and Division of Cancer Sciences, University of Manchester, Manchester, UK \\ Contributions: (I) Conception and design: A Levy, C Faivre-Finn; (II) Administrative support: None; (III) Provision of study materials or patients: A \\ Levy, C Faivre-Finn; (IV) Collection and assembly of data: None; (V) Data analysis and interpretation: None; (VI) Manuscript writing: All authors; \\ (VII) Final approval of manuscript: All authors. \\ Correspondence to: Antonin Levy, MD, PhD. Department of Radiation Oncology, Gustave Roussy, Université Paris-Saclay, F-94805, Villejuif, France. \\ Email: Antonin.LEVY@gustaveroussy.fr.
}

\begin{abstract}
Small-cell lung cancer (SCLC) represents 10-15\% of all lung cancers and has a poor prognosis. Thoracic radiotherapy plays a central role in current SCLC management. Concurrent chemoradiotherapy (CTRT) is the standard of care for localised disease (stage I-III, limited-stage, LS). Definitive thoracic radiotherapy may be offered in metastatic patients (stage IV, extensive stage, ES-SCLC) after chemotherapy. For LS-SCLC, the gold standard is early accelerated hyperfractionated twice-daily CTRT (4 cycles of cisplatin etoposide, starting with the first or second chemotherapy cycle). Modern radiation techniques should be used with involved-field radiotherapy based on baseline CT and PET/CT scans. In ES-SCLC, thoracic radiotherapy should be discussed in cases of initial bulky mediastinal disease/residual thoracic disease not progressing after induction chemotherapy. This strategy was however not assessed in recent trials establishing chemo-immunotherapy as the standard first line treatment in ES-SCLC. Future developments include technical radiotherapy advances and the incorporation of new drugs. Thoracic irradiation is delivered more precisely given technical developments (IMRT, image-guided radiotherapy, stereotactic radiotherapy), reducing the risks of severe adverse events. Stereotactic ablative radiotherapy may be discussed in rare early stage ( $\mathrm{T} 1$ to 2, N0) inoperable patients. A number of current clinical trials are investigating immunoradiotherapy. In this review, we highlight the current role of thoracic radiotherapy and describe ongoing research in the integration of biological surrogate markers, advanced radiotherapy technologies and novel drugs in SCLC patients.
\end{abstract}

Keywords: Thoracic malignancy; radiation oncology; anti-PD(L)1; stereotactic body radiotherapy

Submitted Feb 20, 2020. Accepted for publication Nov 16, 2020.

doi: $10.21037 /$ tlcr-20-305

View this article at: http://dx.doi.org/10.21037/tlcr-20-305

\section{Introduction}

Lung cancer is one of the most common cancers and the leading cause of death from cancer worldwide. Small-cell lung cancer (SCLC) represents $10-15 \%$ of all lung cancers and is characterised by a rapid doubling time and early dissemination (1). In patients without metastatic disease on the initial computed tomography (CT) scan, further staging investigations should include modern imaging such as 18 FDG PET/CT (positron emission tomographycomputed tomography) and brain magnetic resonance imaging (MRI) to detect metastatic disease. Patients with SCLC are typically divided into those with limited-stage (LS, or T1-4 N0-3 M0 according to the $8^{\text {th }}$ lung TNM 
classification) versus extensive-stage (ES; or T1-4 N0-3 M1) disease. Prior to the $8^{\text {th }}$ TNM classification, LS was historically defined as disease confined to one hemithorax (i.e., disease which can be included in a "tolerable" radiation field) (2). About one-third of patients present with LS disease and many have bulky mediastinal disease. Although imaging does not show overt dissemination in the LS setting, the majority of these patients probably already have subclinical metastatic disease. However, local treatments such as thoracic radiotherapy play an important role in patients who have respiratory and general symptoms related to the bulk of thoracic disease. In LS-SCLC, early thoracic radiotherapy delivered concurrently with platinum-based chemotherapy (CTRT) is the cornerstone of treatment (37). In ES-SCLC, initial platinum-based chemotherapy and immunotherapy is the standard treatment $(8,9)$. Thoracic radiotherapy may be subsequently offered in ES-SCLC patients who show a tumour response after initial systemic treatment (10), with the aim of controlling the local disease, palliating symptoms and deferring the need for secondline treatment. In this review, we describe the evidence supporting the use of thoracic radiotherapy in SCLC and discuss current developments and clinical trials in this setting. We present the following article in accordance with the narrative review reporting checklist (available at http:// dx.doi.org/10.21037/tlcr-20-305).

\section{Limited stage (T1-4 NO-3 MO)}

Concurrent thoracic CTRT is the mainstay of treatment for patients with LS (stage I-III: T1-4, N0-3 M0 tumours) with a good performance status (PS) $0-1$. Gold-standard management is early (starting with the first or second cycle of chemotherapy) (11) accelerated hyperfractionated twicedaily (BD) CTRT (4 cycles of cisplatin etoposide) (12-14) with modern radiation techniques [intensity modulated radiotherapy (IMRT); data from non-small cell lung cancer (NSCLC) setting] (15). Post-chemotherapy tumour volume and involved-field radiotherapy should be applied (7). Prophylactic cranial irradiation (PCI) is also recommended for patients without progressive disease after the completion of chemoradiotherapy (described in a separate article of this special edition) (16).

\section{Dose and fractionation}

The current standard of care is BD CTRT, based on two randomized controlled trials that compared once-daily (OD) radiotherapy to 45 Gy over 3 weeks in 1.5 Gy BD, delivered concurrently with cisplatin-etoposide. The first trial (INT 0096) showed the superiority of BD in terms of survival (17) and the second trial (CONVERT) did not show superiority of higher dose OD over BD radiotherapy (12) (Table 1).

The first trial, published in 1999 by Turrisi et al., compared CTRT delivered at a dose of 45 Gy over 3 weeks in 1.5 Gy BD with OD 45 Gy over 5 weeks. Radiotherapy was started at the same time as the first cycle of chemotherapy (17). Staging was performed by CT or MRI of the chest, abdomen, and brain; radionuclide bone scanning; and bilateral iliac-crest bone marrow aspiration and biopsy. All participants $(n=417)$ were to receive four cycles of cisplatin plus etoposide and PCI after CTRT. The median survival was significantly improved in the BD arm (23 vs. 19 months, respectively; $\mathrm{P}=0.004$ ). However, higher toxicities were reported, in particular severe esophagitis leading to hospitalisation. Following the publication of this paper, hyperfractionated radiotherapy was not widely adopted in routine practice due to limitations in the design of the study, logistical issues and an increase in acute toxicity (18).

The second trial, published in 2017 by Faivre-Finn et al., compared BD radiotherapy (45 Gy/30 fractions over 3 weeks) to a higher dose of OD CTRT (66 Gy/33 fractions over 6.5 weeks), starting with the second cycle of chemotherapy, for a total of 4 to 6 cycles of cisplatin plus etoposide (12). Staging included PET-CT (57\%), brain MRI (16\%) and modern radiation therapy techniques (3D conformal radiotherapy, $83.4 \%$; IMRT, 16.6\%) were used. PCI was given if no progression after CTRT ( $82 \%$ of patients) (19). Survival outcomes did not differ between $\mathrm{BD}$ and OD CTRT [median overall survival (OS) $30 \mathrm{vs}$. 25 months, respectively], and was higher than anticipated in both arms. Furthermore, toxicity was similar and lower than expected with both regimens. There was no difference in severe esophagitis between the groups (19\% in both the $\mathrm{BD}$ and OD group). As the trial was designed to show the superiority of OD radiotherapy and was not powered to show equivalence, $\mathrm{BD}$ radiotherapy should continue to be considered the standard of care in this setting.

A recent meta-analysis based on the literature confirmed that BD is better than OD in terms of outcomes (13). A survey of European practice showed that BD radiotherapy delivery increased after the publication of the CONVERT trial (32\% prior to and $42 \%$ after the publication). However, OD radiotherapy remains the most prescribed fractionation. The main reasons for not implementing 
Table 1 Back-to-back comparison of two main phase III trials comparing once-daily to accelerated hyperfractionated twice-daily concurrent radiochemotherapy in LS-SCLC

\begin{tabular}{|c|c|c|}
\hline Study & Turrisi (INT 0096) & Faivre-Finn (CONVERT) \\
\hline Statistics & Superiority (BD exp. arm) & Superiority (OD exp. arm) \\
\hline Baseline staging & $\mathrm{CT}$ or MRI (\%NR), radionuclide bone scanning, bilateral iliac-crest BM sample & CT, PET/CT (57\%), brain MRI (16\%) \\
\hline N pt. & 417 & 547 \\
\hline Chemo. & 4 CDDP Etop & 4-6 CDDP Etop \\
\hline $\mathrm{PCl}$ & If indicated (\% NR) & If indicated (82\%) \\
\hline Median OS & 23 vs. $19 \mathrm{~m}^{\star}, \mathrm{P}=0.004$ & 30 vs. $25 \mathrm{~m}^{\star}$ (NS) \\
\hline 2y PFS & 29 vs. $24 \% *(N S)$ & 40 vs. $38 \% \%^{*}(\mathrm{NS})$ \\
\hline
\end{tabular}

*, BD vs. OD. N pt, number of patients; exp., experimental: CT, computed tomography; MRI, magnetic resonance imaging; PET/CT, positron emission tomography-computed tomography; BM, bone-marrow; RT, radiotherapy; Chemo., chemotherapy; Etop, etoposide; OD, once-daily; BD, twice-daily; m, months; d, days; y, year; NS, not significant; PFS, progression-free survival; OS, overall survival; NR, not reported; LS-SCLC, limited-stage small cell lung cancer.

$\mathrm{BD}$ are logistical issues (44\%), inconvenience for patients (28\%), and the absence of a statistical survival difference between the two arms of the CONVERT trial (which is a misinterpretation given the superiority trial design) (20).

Of note, the effect of dose escalation on locoregional control has been suggested. A combined analysis of 200 patients who received a total dose of 70 Gy suggested a good dose tolerance and comparable efficacy compared to BD radiotherapy (21). A North American intergroup study (CALGB 30610, RTOG 0538, NCT00632853), comparing $45 \mathrm{~Gy} / 30$ fractions BD for 3 weeks to $70 \mathrm{~Gy} / 35$ fractions for 7 weeks is ongoing.

The investigation of hypofractionated radiotherapy is also a topic of interest. A recent phase II study comparing 45 to 60 Gy BD was reported at ASCO and ESMO 2020 in abstract form only. It showed an improvement in 2-year survival but it should be noted that this study is unpublished and included 170 patients (22). These results cannot therefore be considered practice changing and will require confirmation in a phase III study.

\section{Type and timing of radiochemotherapy}

The standard of care consists of four cycles of cisplatin plus etoposide in combination with thoracic radiotherapy, starting with the first or second cycle of chemotherapy $(23,24)$. In case of bulky disease presentation, it may be desirable to achieve tumour response to initial chemotherapy allowing for a decrease in radiation toxicities. In such cases, starting radiotherapy with cycle 3 of chemotherapy is an option, although the data for such an approach is more limited $(25,26)$.

Carboplatin can be used if cisplatin is contraindicated, but this has not been specifically compared to the standard of care of cisplatin in LS-SCLC (27). Additional (e.g., triplet with paclitaxel) (28) or different types of chemotherapy [e.g., irinotecan instead of etoposide, or CEV (cyclophosphamide, epirubicin, and vincristine) $(29,30)]$ did not show improved outcomes when compared to the standard cisplatin etoposide doublet.

Concurrent, sequential and alternating approaches of chemotherapy and thoracic radiotherapy have all been assessed. No survival difference was observed in an EORTC trial comparing alternating or sequential CTRT (cyclophosphamide, doxorubicin, and etoposide) (31). In a more recent study comparing sequential or concurrent CTRT (4 cycles of cisplatin etoposide and 45 Gy BD) on 213 patients with LS-SCLC, a survival improvement was observed in the concurrent arm, although the differences did not reach statistical significance (14). 
In the concurrent setting, it is also suggested that early integration of chemotherapy during thoracic radiotherapy (as compared to delayed thoracic radiotherapy) is associated with improved outcomes. Several randomized trials compared early and late thoracic radiotherapy. Different types of chemotherapy were used, sometimes leading to conflicting results (32). However, in most of the studies that employed the standard platinum-based doublet chemotherapy, early thoracic radiotherapy produced better local control and survival rates than delayed administration $(11,33)$.

\section{Volumes of irradiation}

The standard of care is to use a limited thoracic field of irradiation. Treatment volumes should include the postchemotherapy tumour volume and pre-chemotherapy involved nodal regions (involved-field radiotherapy). Modern imaging, including $\mathrm{PET} / \mathrm{CT}$ and thoracic CT, performed just prior to the first cycle of chemotherapy is therefore crucial to define the target volume. Pathologically confirmed lymph nodes on endoscopic ultrasound with or without fine needle aspiration techniques/mediastinoscopy, if available, should also be included. Smaller fields have led to decreased toxicity without compromising locoregional control rates $(34,35)$. The radiotherapy volume was investigated in an important study by the Southwest Oncology Group (SWOG), which randomized patients after induction chemotherapy to receive irradiation of the post-chemotherapy residue or irradiation of the prechemotherapy volume. There was no difference between the two arms except for an increase in severe toxicity (myelosuppression) for patients irradiated with larger volumes. In addition, local recurrences mainly occurred within the field of radiation and not at the edge of the radiation field for patients with reduced volumes (36). Another prospective randomized trial involving 309 LS SLC patients compared the target volume before and after induction chemotherapy. BD involved-field radiotherapy was applied in both arms and $20 \%$ of patients had a baseline PET/CT. Survival outcomes did not differ between arms, but pre-chemotherapy tumour volume was associated with more G3 esophagitis ( $15.5 \%$ vs. $5.9 \%$; $\mathrm{P}=0.01)(7,37)$. Using pre-chemotherapy volume procedure, only 3 out of 27 patients included in a phase II trial developed an isolated nodal failure (11\%) and all of these were in the ipsilateral supraclavicular region (38). The importance of pre-chemotherapy 18-FDG PET/CT was subsequently highlighted in a prospective study on 60 patients receiving BD involved-field CTRT based on pre-treatment PET/CT. Only 2 isolated nodal failures out of the 60 participants (3\%) were reported (39).

\section{Postoperative thoracic radiotherapy}

In very limited stage I (T1 to 2, N0) LS-SCLC, local treatment with surgery, or stereotactic body radiotherapy (see future directions section) are treatment options if patients are inoperable due to comorbidities. Surgery includes resection of the primary tumour with lobectomy and mediastinal lymph node sampling or dissection. This should then be followed by adjuvant chemotherapy with four cycles of cisplatin-etoposide. There are no clinical trials comparing CTRT vs. surgery.

Postoperative CTRT can be considered in patients who have positive margins or mediastinal lymph node involvement after surgical resection (40-42). However, the data in the LS setting is limited and such recommendations are mostly based on the NSCLC literature. Rare cases of "incidentally resected" (solitary pulmonary nodule) SCLC patients without lymph node sampling may be observed. Given that such patients may be under-staged, and in the absence of adequate lymph node exploration as defined by the IASCLC (43), adjuvant thoracic CTRT can be considered. With regards to treatment volumes in the postoperative setting for SCLC, data is again limited and therefore extrapolation from the NSCLC literature can be considered.

\section{Surgical vs. non-surgical treatment}

Retrospective results from the large US National Cancer Database suggest that surgery may be superior to a nonsurgical approach with CTRT (44-46). Such data should be interpreted with caution given the limitations of this type of retrospective study [selection bias, no progression-free survival (PFS) data]. In the CONVERT trial, LS-SCLC patients with stage I to II (95.3\% stage II) had a median OS of 50 months, which is comparable to surgical series (46).

The NCCN guidelines 2020 (6) state that "patients most likely to benefit from surgery are those with SCLC that is clinical stage I-IIA". In addition, there are no contemporary randomized trials that have compared a surgical to a nonsurgical approach, making the decision process for very early stage SCLC (stage I-II) patients challenging. 


\section{Extensive (M1) stage}

Chemotherapy combined with immunotherapy has become the new systemic standard of care treatment following the results of two randomized phase III trials investigating anti-PD-L1 (atezolizumab or durvalumab) in addition to chemotherapy in ES-SCLC. The role of PCI and thoracic radiotherapy is this setting is controversial $(8,9)$ and beyond the scope of this article.

\section{What role for thoracic radiotherapy?}

In patients with good PS, thoracic radiotherapy may be recommended if, after standard chemotherapy, disease has not progressed but residual thoracic disease is observed. Thoracic radiotherapy following induction chemotherapy was assessed in a randomized phase III trial (10) and in two smaller randomized phase II trials $(33,47)$. In the large CREST EORTC phase III study $(n=498)$, ESSCLC patients in good general condition with any tumour response after four to six cycles of standard chemotherapy (platinum etoposide) were randomized between PCI with or without thoracic irradiation (30 Gy in ten fractions). No difference in one-year OS was observed (primary endpoint; $33 \%$ vs. $28 \%, \mathrm{P}=0.066$ ); but the 2 -year OS (pre-planned analysis) was significantly improved in the thoracic radiotherapy group ( $13 \%$ vs. $3 \% ; \mathrm{P}=0.0004)(10)$. A subsequent analysis of the CREST trial highlighted firstly that the delayed 2-year survival benefit is concordant with the meta-analysis of thoracic radiotherapy for limitedstage SCLC where an increased survival benefit was observed after one year (3); and secondly, that the benefits of thoracic radiotherapy were seen in patients with residual intrathoracic disease but not in those without residual intrathoracic disease. In patients with residual intrathoracic disease, OS was improved in the radiotherapy arm [hazard ratio (HR) 0.81, 95\% CI, 0.66-1.00)], with survival rates at one and two year(s) of $33 \%$ (vs. 26\%) and $12 \%$ (vs. 3\%), respectively (48).

A smaller $(\mathrm{n}=86)$ randomized phase II trial compared PCI +/- consolidative radiation therapy (45 Gy in 15 fractions) to intrathoracic disease and extracranial metastases for ES-SCLC. The study closed early as an interim analysis showed that the futility boundary was crossed for OS. At a median follow-up of 9 months, the one-year OS did not differ between groups but consolidation thoracic radiotherapy resulted in a longer time to progression (47). In an older phase II trial, 210 patients without progression after three cycles of cisplatinetoposide chemotherapy were randomized between BD CTRT [54 Gy/36 fractions over 18 days with carboplatinetoposide followed by two cycles of cisplatin-etoposide $(\mathrm{n}=55)]$ or an additional four cycles of cisplatin-etoposide $(\mathrm{n}=54)$. Better survival rates were observed in the BD CTRT group: median OS 17 vs. 11 months and 5-year survival rate, $9.1 \%$ vs. $3.7 \%$, respectively (49). Higher (>30 Gy/10 fractions) doses of irradiation (OD or BD) have been tested in this setting, although no strong data support this strategy $(47,50)$.

Generally, radiotherapy (30 Gy/10 fractions based on CREST trial) after induction chemotherapy may be offered in fit patients with limited extrathoracic tumour burden and initial bulky disease with either a complete extrathoracic response or partial thoracic response (51). It should also be noted that recent trials evaluating front-line chemotherapy and immunotherapy in the stage IV setting did not involve thoracic radiation $(8,9)$. The role of thoracic radiotherapy in the era of immunotherapy in this setting is therefore not well defined.

\section{Palliative thoracic radiotherapy}

Palliative thoracic radiotherapy plays a role in symptom management in patients with SCLC. Generally, chemotherapy/immunotherapy is offered as frontline treatment as most patients experience a rapid response to systemic therapy. However, in the case of symptoms related to residual thoracic disease (such as cough, dyspnoea, haemoptysis) or pain related to direct involvement of the chest wall or vertebral bodies, palliative radiotherapy may be used. Palliative radiotherapy may also be used in case of superior vena cava obstruction (in conjunction with systemic therapy), as it can achieve complete relief of symptoms within two weeks in most SCLC patients (52).

\section{Future directions}

Recently, efforts have been made to integrate tumour biology, newer technologies and novel drugs in the management of SCLC patients. The main strategies are summarised below.

\section{Technical improvements in thoracic radiation oncology}

Recent technical developments have enabled the delivery of more precise thoracic irradiation, resulting in reduction 
of dose delivered to organs at risk (OARs). IMRT is a technique that adds fluence modulation to beam shaping, which improves radiotherapy dose conformity around the tumour and spares surrounding normal structures $(53,54)$. Secondary analysis from a phase III trial assessing dose-escalation radiotherapy in locally advanced NSCLC showed that patients treated with IMRT had significantly less G3-5 pneumonitis and lower heart doses (15). Limited data on IMRT is available in the SCLC setting. A study from the MD Anderson Cancer Center compared the outcome of 119 SCLC patients receiving 3DCRT and 104 receiving IMRT. OS and disease-free survival were comparable in both groups in multivariable and propensity score-matched analyses. However, IMRT patients required significantly fewer percutaneous feeding tube insertions (5\% vs. 17\%) (55).

New imaging methods, mainly on-board cone beam CT scan, incorporated into the linear accelerator, allows more accurate set-up and therefore improves the accuracy of the radiation beams ("image-guided" radiotherapy). MRIguided linear accelerators are also becoming available. Such techniques could permit improved target visualization "beam-on" and facilitates the investigation of daily online plan adaptation and personalised dose intensification without exposing patients to ionising radiation (56).

Proton beam therapy is an advanced radiation treatment characterised by its Bragg peak, whereby a high dose is delivered to the target, followed by an immediate drop in energy resulting in minimal to no exit dose compared to standard photon radiotherapy. Given this unique physical property, proton therapy may increase the therapeutic ratio and allow more critical structures to be spared. Proton-beam therapy was evaluated in a prospective study including 30 LS-SCLC patients receiving (OD or BD) CTRT. As compared to IMRT plans, protons allowed reductions in several organs' mean doses (spinal cord, heart, and lung) but not in the oesophageal mean dose or the lung volume receiving at least $20 \mathrm{~Gy}(\mathrm{~V} 20)$. Grade 4 esophagitis $(\mathrm{n}=1)$, grade 3 pneumonitis $(n=1)$, anorexia $(n=1)$, and pericardial effusion ( $\mathrm{n}=1$ ) were observed (57). A phase II randomized trial compared passive scattering proton therapy versus IMRT, both with concurrent chemotherapy, in 147 inoperable NSCLC patients. Protons exposed less heart tissue at all dose levels. Grade $\geq 3$ radiation pneumonitis and local failure (co-primary endpoints) were similar in both arms (58). Since this publication, newer protons planning techniques have become available (e.g., proton intensity modulation and pencil beam scanning) and have the potential to improve the therapeutic ratio of protons $v s$. photons (59). Several studies comparing protons to conventional photon techniques are ongoing for stage III NSCLC (NCT01993810, NCT02731001), but no trials are ongoing in SCLC.

\section{SBRT in stage I SCLC}

Stereotactic radiotherapy allows the administration of socalled "ablative" doses to early-stage lung lesions with very steep dose fall-off. SBRT technique has become the standard treatment for peripheral medically non-operable patients with early stage NSCLC. Retrospective studies have shown high local control rate (85-95\%), and a low toxicity profile $(60,61)$. Guidelines on the treatment of early stage NSCLC with SBRT have been published and should be applied in patients with SCLC treated with SBRT (62). Stage I (T1 to 2, N0) SCLC presentation is uncommon but in medically inoperable cases or in patients refusing surgery, thoracic SBRT may play a role. As described earlier, in the absence of mediastinal node sampling, chemotherapy should also be offered in this setting. There is currently no prospective data on such a strategy. Retrospective data from a large national database suggests that stage I SCLC treated with SBRT regimens incorporating chemotherapy had comparable outcomes to those treated with CTRT $(63,64)$. A multicentre analysis of 2107 stage I SCLC compared outcomes of patients who received SBRT/chemotherapy (7\%) and OD CTRT (93\%). SBRT was delivered at a dose (interquartile range) of 48-54 Gy in 3-5 fractions. OS did not differ by radiotherapy technique in multivariable and propensity score-matched analyses (64). Such data should however be interpreted with extreme caution given their inherent limitations, which include their retrospective nature, selection bias, and a lack of PFS data.

\section{Immune-checkpoint inbibitors (ICI)}

Following the success of the PACIFIC study in stage 3 NSCLC, several trials are ongoing to assess the role of ICI in SCLC both in the consolidation setting and the concurrent setting (Table 2). In LS-SCLC, the STIMULI, ADRIATIC, and ACHILES trials are assessing consolidation immunotherapy after thoracic CTRT and PCI. The ADRIATIC study (NCT03703297) is a randomized phase II trial comparing durvalumab (anti PDL1 until disease progression) +/- tremelimumab (antiCTLA4, for 4 cycles) or placebo as consolidation therapy 
Table 2 Ongoing trials evaluating immunotherapy in SCLC patients receiving thoracic radiotherapy

\begin{tabular}{|c|c|c|c|c|c|}
\hline $\mathrm{ICl}$ timing with TRT & Drug & TRT (Gy) & Phase & Main result & Name, NCT number \\
\hline Consolidation & Durvalumab \pm tremelimumab & OD/BD & 3 & Ongoing & ADRIATIC NCT03703297 (65) \\
\hline Consolidation & Nivolumab + ipilimumab & OD/BD & 2 & Ongoing & STIMULI NCT02046733 \\
\hline Consolidation & Atezolizumab & BD & 2 & Ongoing & ACHILES NCT03540420 \\
\hline Concurrent + consolidation & Durvalumab & $\begin{array}{c}52.2 \\
(2.1 \mathrm{~Gy} / \mathrm{Fx})\end{array}$ & 2 & Ongoing & NCT03585998 \\
\hline \multicolumn{6}{|l|}{ Extensive-stage (M1) } \\
\hline Concurrent + consolidation & Pembrolizumab & 45 & 1 & No DLT & NCT02402920 (66) \\
\hline
\end{tabular}

OD: 60 to 66 Gy within 6 weeks (standard once-daily schedule); BD: 45 Gy within 3 weeks (standard twice-daily schedule). DLT, dose limiting-toxicity; PFS, progression-free survival; ICI, immune checkpoint inhibitors; TRT, thoracic radiation therapy; fx, fraction.

for patients with LS-SCLC without disease progression after CTRT (65). The STIMULI randomized phase II trial (NCT02046733) compares consolidation nivolumab (anti PD-1, for one year) + ipilimumab (anti-CTLA4, for 4 cycles) $v s$. no further treatment (closed to accrual in 2019). Another randomized phase II study is comparing atezolizumab with no further treatment after CTRT in LS-SCLC (ACHILES, NCT03540420). A further study [NRG Oncology/Alliance LU005 phase II/III trial (NCT03811002)] is investigating atezolizumab delivered concurrently with CTRT and in the consolidation setting in LS-SCLC patients. A non-randomized phase II study is evaluating concurrent CTRT + durvalumab, and followed by consolidation durvalumab (NCT03585998).

In ES-SCLC, a phase I trial (NCT02402920) assessed pembrolizumab concurrently with thoracic irradiation (45 Gy in 15 daily fractions) after induction chemotherapy. Pembrolizumab is continued for up to 16 cycles after radiotherapy. No dose-limiting toxicity was observed at the three-dose level of pembrolizumab (100 to 200) and $2(6 \%)$ patients experienced grade 3 events that were unlikely/doubtfully related to protocol therapy. Median PFS and OS were 6.1 and 8.4 months but should be interpreted with caution given the limited sample size (66). Another single arm phase I/II trial studied nivolumab (for one year) + ipilimumab (for 4 cycles) two weeks after consolidative thoracic radiotherapy (30 Gy/10 fractions).
This study recruited 21 of the 52 planned patients, and was discontinued early due to a planned interim analysis showing that PFS was low compared to historic estimates (6-month PFS of $24 \%$ and median PFS of 4.5 months). The toxicity profile was consistent with the known adverse events attributable to the ICI combination (NCT03043599) (67). A phase I trial is testing durvalumab +/- tremelimumab or olaparib (4 cycles), delivered concurrently with thoracic radiotherapy (30 Gy/10 fractions) after platinum-based chemotherapy in ES-SCLC patients (NCT03923270). Durvalumab is continued for up to 13 cycles after completion of radiotherapy.

\section{Conclusions}

Thoracic radiotherapy plays an important role in the management of SCLC. CTRT with twice-daily radiotherapy (Table 1) is the cornerstone of LS-SCLC management and thoracic radiotherapy should be considered in ES-SCLC with residual thoracic disease after chemotherapy. Advanced thoracic radiotherapy techniques and technologies are available with the integration of PET/ CT and 4DCT for planning, the use of IMRT and omission of elective nodal irradiation and the availability of cone beam CT for treatment verification. Immunoradiotherapy has become standard of care in the ES setting and many ongoing trials are evaluating the integration of ICI with 
CTRT in the LS setting (Table 2).

New biological surrogate markers are still needed to better select patients who could benefit from these promising but expensive treatments. Circulating tumour cells (CTC) are known to be prognostic biomarkers in SCLC patients and may correlate with tumour response during treatment $(68,69)$. The challenge now is to design trials to demonstrate the clinical benefit of a biomarkerdriven strategy in the SCLC.

Commonly occurring mutations in SCLC (TP53, $R B 1$, multiple epigenetic regulators, and Notch family members) are all loss-of-function, which limits the possibility of targeting oncogenic mutations (70). Four recently discovered key transcriptional regulators may offer therapeutic opportunities: ASCL1, NEUROD1, POU2F3, and YAP1 (71). Several other candidate immune biomarkers could be tested for patient selection in the era of personalized (immuno)radiotherapy (72). Such efforts to integrate tumour biology, newer technologies and novel drugs will hopefully lead to the improvement of the therapeutic index of radiotherapy (73) in SCLC patients.

\section{Acknowledgments}

The authors would like to acknowledge Dr. Kate Wicks for editorial assistance.

Funding: None.

\section{Footnote}

Provenance and Peer Review: This article was commissioned by the Guest Editors (Jacek Jassem and Rafal Dziadziuszko) for the series "Radiotherapy in thoracic malignancies" published in Translational Lung Cancer Research. The article has undergone external peer review.

Reporting Checklist: The authors have completed the narrative review reporting checklist. Available at http:// dx.doi.org/10.21037/tlcr-20-305

Conflicts of Interest: All authors have completed the ICMJE uniform disclosure form (available at http://dx.doi. org/10.21037/tlcr-20-305). The series "Radiotherapy in thoracic malignancies" was commissioned by the editorial office without any funding or sponsorship. Dr. CFF reports grants from Merck, grants from AstraZeneca, grants from Elekta, grants from Pfizer, outside the submitted work. The authors have no other conflicts of interest to declare.
Ethical Statement: The authors are accountable for all aspects of the work in ensuring that questions related to the accuracy or integrity of any part of the work are appropriately investigated and resolved.

Open Access Statement: This is an Open Access article distributed in accordance with the Creative Commons Attribution-NonCommercial-NoDerivs 4.0 International License (CC BY-NC-ND 4.0), which permits the noncommercial replication and distribution of the article with the strict proviso that no changes or edits are made and the original work is properly cited (including links to both the formal publication through the relevant DOI and the license). See: https://creativecommons.org/licenses/by-nc-nd/4.0/.

\section{References}

1. Cancer Today [Internet]. [cited 2020 Feb 17]. Available online: https://gco.iarc.fr/today/home

2. Roswit B, Patno ME, Rapp R, et al. The survival of patients with inoperable lung cancer: a large-scale randomized study of radiation therapy versus placebo. Radiology 1968;90:688-97.

3. Pignon JP, Arriagada R, Ihde DC, et al. A Meta-Analysis of Thoracic Radiotherapy for Small-Cell Lung Cancer. N Engl J Med 1992;327:1618-24.

4. Warde P, Payne D. Does thoracic irradiation improve survival and local control in limited-stage small-cell carcinoma of the lung? A meta-analysis. J Clin Oncol 1992;10:890-5.

5. Früh M, De Ruysscher D, Popat S, et al. Small-cell lung cancer (SCLC): ESMO Clinical Practice Guidelines for diagnosis, treatment and follow-up. Ann Oncol 2013;24:vi99-105.

6. Enhanced Reader [Internet]. [cited 2020 Feb 17]. Available online: moz-extension://7584cb4065cf-4e9a-bf43-20851612e5f2/enhanced-reader. html?openApp\&pdf=https\%3A\%2F\%2Fw ww.nccn.org\%2Fprofessionals\%2Fphysician_ gls $\% 2$ Fpdf\% 2Fsclc.pdf

7. Le Pechoux C, Faivre-Finn C, Ramella S, et al. ESTRO ACROP guidelines for target volume definition in the thoracic radiation treatment of small cell lung cancer. Radiother Oncol 2020;152:89-95.

8. Horn L, Mansfield AS, Szczęsna A, et al. First-Line Atezolizumab plus Chemotherapy in Extensive-Stage Small-Cell Lung Cancer. N Engl J Med 2018;379:2220-9.

9. Paz-Ares L, Dvorkin M, Chen Y, et al. Durvalumab plus 
platinum-etoposide versus platinum-etoposide in firstline treatment of extensive-stage small-cell lung cancer (CASPIAN): a randomised, controlled, open-label, phase 3 trial. Lancet 2019;394:1929-39.

10. Slotman BJ, van Tinteren H, Praag JO, et al. Use of thoracic radiotherapy for extensive stage small-cell lung cancer: a phase 3 randomised controlled trial. Lancet 2015;385:36-42.

11. De Ruysscher D, Pijls-Johannesma M, Bentzen SM, et al. Time Between the First Day of Chemotherapy and the Last Day of Chest Radiation Is the Most Important Predictor of Survival in Limited-Disease Small-Cell Lung Cancer. J Clin Oncol 2006;24:1057-63.

12. Faivre-Finn C, Snee M, Ashcroft L, et al. Concurrent once-daily versus twice-daily chemoradiotherapy in patients with limited-stage small-cell lung cancer (CONVERT): an open-label, phase 3, randomised, superiority trial. Lancet Oncol 2017;18:1116-25.

13. Wu Q, Xiong Y, Zhang S, et al. A Meta-Analysis of the Efficacy and Toxicity of Twice-Daily vs. Once-Daily Concurrent Chemoradiotherapy for Limited-Stage Small Cell Lung Cancer Based on Randomized Controlled Trials. Front Oncol 2020;9:1460.

14. Takada M, Fukuoka M, Kawahara M, et al. Phase III Study of Concurrent Versus Sequential Thoracic Radiotherapy in Combination With Cisplatin and Etoposide for Limited-Stage Small-Cell Lung Cancer: Results of the Japan Clinical Oncology Group Study 9104. J Clin Oncol 2002;20:3054-60.

15. Chun SG, Hu C, Choy H, et al. Impact of IntensityModulated Radiation Therapy Technique for Locally Advanced Non\{ \textendash\}Small-Cell Lung Cancer: A Secondary Analysis of the $\{\mathrm{NRG}\}$ Oncology \{RTOG\} 0617 Randomized Clinical Trial. J Clin Oncol 2017;35:56-62.

16. Aupérin A, Arriagada R, Pignon JP, et al. Prophylactic cranial irradiation for patients with small-cell lung cancer in complete remission. Prophylactic Cranial Irradiation Overview Collaborative Group. N Engl J Med 1999;341:476-84.

17. Turrisi AT, Kim K, Blum R, et al. Twice-daily compared with once-daily thoracic radiotherapy in limited smallcell lung cancer treated concurrently with cisplatin and etoposide. N Engl J Med 1999;340:265-71.

18. Farrell MJ, Yahya JB, Degnin C, et al. Radiation Dose and Fractionation for Limited-stage Small-cell Lung Cancer: Survey of US Radiation Oncologists on Practice Patterns. Clin Lung Cancer 2019;20:13-9.

19. Levy A, Le Péchoux C, Mistry H, et al. Prophylactic
Cranial Irradiation for Limited-Stage Small-Cell Lung Cancer Patients: Secondary Findings From the Prospective Randomized Phase 3 CONVERT Trial. J Thorac Oncol 2019;14:294-7.

20. Levy A, Hendriks LEL, Le Péchoux C, et al. Current management of limited-stage SCLC and CONVERT trial impact: Results of the EORTC Lung Cancer Group survey. Lung Cancer 2019;136:145-7.

21. Salama JK, Hodgson L, Pang H, et al. A pooled analysis of limited-stage small-cell lung cancer patients treated with induction chemotherapy followed by concurrent platinumbased chemotherapy and 70 Gy daily radiotherapy: CALGB 30904. J Thorac Oncol 2013;8:1043-9.

22. Gronberg BH, Killingberg KT, Fløtten $\varnothing$, et al. Randomized phase II trial comparing the efficacy of standard-dose with high-dose twice-daily thoracic radiotherapy (TRT) in limited disease small-cell lung cancer (LD SCLC). J Clin Oncol 2020;38:9007.

23. Fried DB, Morris DE, Poole C, et al. Systematic Review Evaluating the Timing of Thoracic Radiation Therapy in Combined Modality Therapy for Limited-Stage SmallCell Lung Cancer. J Clin Oncol 2004;22:4837-45.

24. Pijls-Johannesma M, De Ruysscher D, Vansteenkiste J, et al. Timing of chest radiotherapy in patients with limited stage small cell lung cancer: A systematic review and metaanalysis of randomised controlled trials. Cancer Treat Rev 2007;33:461-73.

25. Skarlos DV, Samantas E, Briassoulis E, et al. Randomized comparison of early versus late hyperfractionated thoracic irradiation concurrently with chemotherapy in limited disease small-cell lung cancer: A randomized phase II study of the Hellenic Cooperative Oncology Group (HeCOG). Ann Oncol 2001;12:1231-8.

26. Sun JM, Ahn YC, Choi EK, et al. Phase III trial of concurrent thoracic radiotherapy with either first- or third-cycle chemotherapy for limited-disease small-cell lung cancer. Ann Oncol 2013;24:2088-92.

27. Rossi A, Di Maio M, Chiodini P, et al. Carboplatin- or Cisplatin-Based Chemotherapy in First-Line Treatment of Small-Cell Lung Cancer: The COCIS Meta-Analysis of Individual Patient Data. J Clin Oncol 2012;30:1692-8.

28. Horn L, Bernardo P, Sandler A, et al. A phase II study of paclitaxel + etoposide + cisplatin + concurrent radiation therapy for previously untreated limited stage small cell lung cancer (E2596): a trial of the Eastern Cooperative Oncology Group. J Thorac Oncol 2009;4:527-33.

29. Kubota K, Hida T, Ishikura S, et al. Etoposide and cisplatin versus irinotecan and cisplatin in patients with 
limited-stage small-cell lung cancer treated with etoposide and cisplatin plus concurrent accelerated hyperfractionated thoracic radiotherapy (JCOG0202): a randomised phase 3 study. Lancet Oncol 2014;15:106-13.

30. Sundstrøm S, Bremnes RM, Kaasa S, et al. Cisplatin and Etoposide Regimen Is Superior to Cyclophosphamide, Epirubicin, and Vincristine Regimen in Small-Cell Lung Cancer: Results From a Randomized Phase III Trial With 5 Years' Follow-Up. J Clin Oncol 2002;20:4665-72.

31. Gregor A, Drings P, Burghouts J, et al. Randomized trial of alternating versus sequential radiotherapy/chemotherapy in limited-disease patients with small-cell lung cancer: a European Organization for Research and Treatment of Cancer Lung Cancer Cooperative Group Study. J Clin Oncol 1997;15:2840-9.

32. Spiro SG, James LE, Rudd RM, et al. Early Compared With Late Radiotherapy in Combined Modality Treatment for Limited Disease Small-Cell Lung Cancer: A London Lung Cancer Group Multicenter Randomized Clinical Trial and Meta-Analysis. J Clin Oncol 2006;24:3823-30.

33. Jeremic B, Shibamoto Y, Acimovic L, et al. Initial versus delayed accelerated hyperfractionated radiation therapy and concurrent chemotherapy in limited small-cell lung cancer: a randomized study. J Clin Oncol 1997;15:893-900.

34. Arriagada R, Pellae-Cosset B, Cueto Ladron de Guevara $\mathrm{J}$, et al. Alternating radiotherapy and chemotherapy schedules in limited small cell lung cancer: analysis of local chest recurrences. Radiother Oncol 1991;20:91-8.

35. Laurans M, Botticella A, Moukasse Y, et al. Cancer du poumon et irradiation ganglionnaire prophylactique : un débat clos? Cancer/Radiothérapie 2019;23:701-7.

36. Liengswangwong V, Bonner JA, Shaw EG, et al. Limitedstage small-cell lung cancer: patterns of intrathoracic recurrence and the implications for thoracic radiotherapy. J Clin Oncol 1994;12:496-502.

37. $\mathrm{Hu} \mathrm{X}, \mathrm{Bao} \mathrm{Y}, \mathrm{Xu} \mathrm{Y}$, et al. Final report of a prospective randomized study on thoracic radiotherapy target volume for limited stage small cell lung cancer with radiation dosimetric analyses. Cancer 2020;126:840-9.

38. De Ruysscher D, Bremer RH, Koppe F, et al. Omission of elective node irradiation on basis of CT-scans in patients with limited disease small cell lung cancer: A phase II trial. Radiother Oncol 2006;80:307-12.

39. van Loon J, De Ruysscher D, Wanders R, et al. Selective Nodal Irradiation on Basis of 18FDG-PET Scans in Limited-Disease Small-Cell Lung Cancer: A Prospective Study. Int J Radiat Oncol Biol Phys 2010;77:329-36.
40. Putora PM, De Ruysscher D, Glatzer M, et al. The role of postoperative thoracic radiotherapy and prophylactic cranial irradiation in early stage small cell lung cancer: Patient selection among ESTRO experts. Radiother Oncol 2020;145:45-8.

41. Wakeam E, Giuliani M, Leighl NB, et al. Indications for Adjuvant Mediastinal Radiotherapy in Surgically Resected Small Cell Lung Cancer. Ann Thorac Surg 2017;103:1647-53.

42. Wong AT, Rineer J, Schwartz D, et al. Assessing the Impact of Postoperative Radiation Therapy for Completely Resected Limited-Stage Small Cell Lung Cancer Using the National Cancer Database. J Thorac Oncol 2016;11:242-8.

43. Rami-Porta R, Wittekind C, Goldstraw P. Complete resection in lung cancer surgery: proposed definition. Lung Cancer 2005;49:25-33.

44. Wakeam E, Acuna SA, Leighl NB, et al. Surgery Versus Chemotherapy and Radiotherapy For Early and Locally Advanced Small Cell Lung Cancer: A Propensity-Matched Analysis of Survival. Lung Cancer 2017;109:78-88.

45. Uprety D, Arjyal L, Vallatharasu Y, et al. Utilization of Surgery and Its Impact on Survival in Patients With Early Stage Small-cell Lung Cancer in the United States. Clin Lung Cancer 2020;21:186-93.e2.

46. Salem A, Mistry H, Hatton M, et al. Association of Chemoradiotherapy With Outcomes Among Patients With Stage I to II vs Stage III Small Cell Lung Cancer: Secondary Analysis of a Randomized Clinical Trial. JAMA Oncol 2019;5:e185335.

47. Gore EM, Hu C, Sun AY, et al. Randomized Phase II Study Comparing Prophylactic Cranial Irradiation Alone to Prophylactic Cranial Irradiation and Consolidative Extracranial Irradiation for Extensive-Disease Small Cell Lung Cancer (ED SCLC): NRG Oncology RTOG 0937. J Thorac Oncol 2017;12:1561-70.

48. Slotman BJ, van Tinteren H, Praag JO, et al. Radiotherapy for extensive stage small-cell lung cancer - Authors' reply. Lancet 2015;385:1292-3.

49. Jeremic B, Shibamoto Y, Nikolic N, et al. Role of Radiation Therapy in the Combined-Modality Treatment of Patients With Extensive Disease Small-Cell Lung Cancer: A Randomized Study. J Clin Oncol 1999;17:2092.

50. Li QW, Qiu B, Wang B, et al. Comparison of hyper- and hypofractionated radiation schemes with IMRT technique in small cell lung cancer: Clinical outcomes and the introduction of extended LQ and TCP models. Radiother Oncol 2019;136:98-105. 
51. Putora PM, Glatzer M, De Ruysscher D, et al. Consolidative thoracic radiotherapy in stage IV small cell lung cancer: Selection of patients amongst European IASLC and ESTRO experts. Radiother Oncol 2019;135:74-7.

52. Rodrigues G, Videtic GMM, Sur R, et al. Palliative thoracic radiotherapy in lung cancer: An American Society for Radiation Oncology evidence-based clinical practice guideline. Pract Radiat Oncol 2011;1:60-71.

53. Brown S, Banfill K, Aznar MC, et al. The evolving role of radiotherapy in non-small cell lung cancer. Br J Radiol 2019;92:20190524.

54. Chan C, Lang S, Rowbottom C, et al. Intensity-Modulated Radiotherapy for Lung Cancer: Current Status and Future Developments. J Thorac Oncol 2014;9:1598-608.

55. Shirvani SM, Juloori A, Allen PK, et al. Comparison of 2 Common Radiation Therapy Techniques for Definitive Treatment of Small Cell Lung Cancer. Int J Radiat Oncol Biol Phys 2013;87:139-47.

56. Bainbridge H, Salem A, Tijssen RHN, et al. Magnetic resonance imaging in precision radiation therapy for lung cancer. Transl Lung Cancer Res 2017;6:689-707.

57. Rwigema JM, Verma V, Lin L, et al. Prospective study of proton-beam radiation therapy for limited-stage small cell lung cancer. Cancer 2017;123:4244-51.

58. Liao Z, Lee JJ, Komaki R, et al. Bayesian Adaptive Randomization Trial of Passive Scattering Proton Therapy and Intensity-Modulated Photon Radiotherapy for Locally Advanced Non-Small-Cell Lung Cancer. J Clin Oncol 2018;36:1813-22.

59. Troost EGC, Wink KCJ, Roelofs E, et al. Photons or protons for reirradiation in (non-)small cell lung cancer: Results of the multicentric ROCOCO in silico study. Br J Radiol 2020;93:20190879.

60. Loganadane G, Martinetti F, Mercier O, et al. Stereotactic ablative radiotherapy for early stage non-small cell lung cancer: A critical literature review of predictive factors of relapse. Cancer Treat Rev 2016;50:240-6.

61. Ball D, Mai GT, Vinod S, et al. Stereotactic ablative radiotherapy versus standard radiotherapy in stage 1 nonsmall-cell lung cancer ( $\{$ TROG\} 09.02 \{CHISEL\}): a phase 3 , open-label, randomised controlled trial. Lancet Oncol 2019;20:494-503.
62. Guckenberger M, Andratschke N, Dieckmann K, et al. ESTRO ACROP consensus guideline on implementation and practice of stereotactic body radiotherapy for peripherally located early stage non-small cell lung cancer. Radiother Oncol 2017;124:11-7.

63. Rathod S, Koul R, Bashir B, et al. Role of Stereotactic Body Radiation Therapy in Early Stage Small Cell Lung Cancer in the Era of Lung Cancer Screening. Am J Clin Oncol 2019;42:123-30.

64. Verma V, Hasan S, Wegner RE, et al. Stereotactic ablative radiation therapy versus conventionally fractionated radiation therapy for stage I small cell lung cancer. Radiother Oncol 2019;131:145-9.

65. Senan S, Okamoto I, Lee GW, et al. Design and Rationale for a Phase III, Randomized, Placebo-controlled Trial of Durvalumab With or Without Tremelimumab After Concurrent Chemoradiotherapy for Patients With Limited-stage Small-cell Lung Cancer: The ADRIATIC Study. Clin Lung Cancer 2020;21:e84-8.

66. Welsh JW, Heymach JV, Chen D, et al. Phase I Trial of Pembrolizumab and Radiation Therapy after Induction Chemotherapy for Extensive-Stage Small Cell Lung Cancer. J Thorac Oncol 2020;15:266-73.

67. Perez BA, Kim S, Dilling TJ, et al. A Prospective Single Arm Phase I/II Study: Consolidative Ipilimumab and Nivolumab with Thoracic Radiotherapy after Platinum Based Chemotherapy for Patients with Extensive-Stage Small Cell Lung Cancer. Int J Radiat Oncol 2019;105:S36.

68. Tay RY, Fernández-Gutiérrez F, Foy V, et al. Prognostic value of circulating tumour cells in limited-stage smallcell lung cancer: analysis of the concurrent once-daily versus twice-daily radiotherapy (CONVERT) randomised controlled trial. Ann Oncol 2019;30:1114-20.

69. Hou JM, Krebs MG, Lancashire L, et al. Clinical Significance and Molecular Characteristics of Circulating Tumor Cells and Circulating Tumor Microemboli in Patients With Small-Cell Lung Cancer. J Clin Oncol 2012;30:525-32.

70. George J, Lim JS, Jang SJ, et al. Comprehensive genomic profiles of small cell lung cancer. Nature 2015;524:47-53.

71. Rudin CM, Poirier JT, Byers LA, et al. Molecular subtypes of small cell lung cancer: a synthesis of human and mouse model data. Nat Rev Cancer 2019;19:289-97. 
72. Levy A, Nigro G, Sansonetti PJ, et al. Candidate immune biomarkers for radioimmunotherapy. Biochim Biophys Acta Rev Cancer 2017;1868:58-68.

Cite this article as: Levy A, Botticella A, Le Péchoux C, Faivre-Finn C. Thoracic radiotherapy in small cell lung cancer-a narrative review. Transl Lung Cancer Res 2021;10(4):2059-2070. doi: 10.21037/tlcr-20-305
73. Chargari C, Magne N, Guy JB, et al. Optimize and refine therapeutic index in radiation therapy: Overview of a century. Cancer Treat Rev 2016;45:58-67. 